\title{
Finite-size scaling for discontinuous nonequilibrium phase transitions
}

\author{
Marcelo M. de Oliveira ${ }^{1}$, M. G. E. da Luz ${ }^{2}$ and Carlos E. Fiore ${ }^{3}$ \\ ${ }^{1}$ Departamento de Física e Matemática, CAP, Universidade Federal de São João del Rei, 36420-000 Ouro Branco-MG, Brazil, \\ 2 Departamento de Física, Universidade Federal do Paraná, 81531-980 Curitiba-PR, Brazil \\ ${ }^{3}$ Instituto de Física, Universidade de São Paulo, 05315-970 São Paulo-SP, Brazil
}

(Dated: April 3, 2018)

\begin{abstract}
A finite size scaling theory, originally developed only for transitions to absorbing states [Phys. Rev. E 92, 062126 (2015)], is extended to distinct sorts of discontinuous nonequilibrium phase transitions. Expressions for quantities such as, response functions, reduced cumulants and equal area probability distributions, are derived from phenomenological arguments. Irrespective of system details, all these quantities scale with the volume, establishing the dependence on size. The approach generality is illustrated through the analysis of different models. The present results are a relevant step in trying to unify the scaling behavior description of nonequilibrium transition processes.
\end{abstract}

Methodologically speaking, nonequilibrium bears a relation to equilibrium phase transitions somewhat similar to that between nonlinear and linear dynamical systems. Indeed, well established theoretical frameworks, universal methods of analysis and generic efficient calculation protocols are far better developed for the latter than for the former. Yet, nonequilibrium are conceivably more prevalent than equilibrium transitions, ubiquitous in such diverse phenomena [1 5] as, interface growth, epidemics, chemical reactions, population dynamics, flow in biological systems [6], spatio-temporal chaos in liquid crystals [7], behavior of driven suspensions [8], and superconducting vortices [9] (see [10] for a survey).

Most of the studies on nonequilibrium has been directed to universality and scaling in the continuous context, with much less attention being paid to discontinuous nonequilibrium phase transitions (DNPT) [11, 12]. Despite this, DNPT are quite commonly observed in problems like heterogeneous catalysis [13, 14], ecological processes [15, 16], granular systems [17], replicator dynamics [18], cooperative coinfection [19], language formation [20] and social influence [21].

There are few general results for nonequilibrium phase transitions [2, 3, 22], one example being the celebrated Jarzynski equality [23] for free energy differences $\Delta F$. Another, for DNPT, is that to emerge they seem to require an effective mechanism suppressing the formation of minority islands induced by fluctuations 24, 25]. Also, DNPT allow a finite-size scaling (FSS). But this fact has been shown only for transitions to absorbing states [12], based on the idea of quasi-stationary (QS) ensembles 26, 27]. Calculations for several cases in $d$ dimensions reveal that distinct QS quantities scale with the system volume $V=L^{d}$. Further, the shift in the coexistence point value goes with $1 / V$. Such findings are in great similarity with equilibrium phase transitions [28 35], whose coexistence points correspond to equal $F$ 's in the distinct phases and for the $F$ 's second derivatives displaying a $\delta$ function-like shape in the thermodynamic limit.

Here we extend the results in [12] for systems admitting nonequilibrium steady states (without exhibiting ab- sorbing states). Relying on solid phenomenological arguments, we deduce a general FSS behavior for DNPT. We determine that different quantities, like response functions, reduced ratio cumulants and probability distributions, go with the inverse of the system volume. We illustrate our method addressing diverse nonequilibrium models, as absorption-desorption on catalytic surfaces, systems with up-down $Z_{2}$ symmetry, and competitive interactions in bipartite sublattices, all them displaying distinct features and symmetries. Our approach unveils an universal scaling behavior for DNPT in close analogy with the equilibrium case. Hence, it constitutes an additional example of the unexpected resemblance between procedures treating certain aspects of equilibrium and nonequilibrium thermodynamics [12, 36].

Our starting point is that in some relevant aspects equilibrium and nonequilibrium phase transitions are akin when the latter present stationary states 37]. For instance, as very clearly put in [38], for a sufficiently large variation of a control parameter $\lambda$, DNPT can be viewed as the passage through distinct steady states, each typified by the value of an order parameter $m$. Moreover - at least regarding $m$ - these states must be stable against 22] external disturbances, fluctuations, and eventual internal currents (typically arising when the detailed balance is not observed).

The above is the case when the net probability current (yielding the order parameter time evolution) in each steady state is null, i.e., if the microscopic dynamics always obeys the global balance, namely [39],

$$
\sum_{y} p(x) w(x \rightarrow y)=\sum_{y} p(y) w(y \rightarrow x)
$$

for $x, y$ micro-configurations associated to $m, w(x \rightarrow y)$ their transition rate, and $p(z)$ the probability of $z$. Therefore, in such situation we can ascribe a stable probability density to the nonequilibrium steady state [40]. Also, its stationarity allied to the global balance support an extended version of the central limit theorem for the distribution of $m$, given by Gaussians [41], even in the nonequilibrium case [36]. 
Thus, in the vicinity of the coexistence at $\lambda_{0}$ (i.e., for $\Delta \lambda=\lambda-\lambda_{0}$ small) and for a finite but large enough volume $V$, the phase $C$ probability distribution $P_{V}^{(C)}(m)$ should read (up to a normalization constant, see below)

$$
P_{V}^{(C)}(m)=\sqrt{\frac{g(V)}{2 \pi}} \exp \left[g(V)\left(\Delta \lambda m-\frac{\left(m-m_{C}\right)^{2}}{2 \chi_{C}}\right)\right] .
$$

Here $\chi_{C}$ is the variance and $g(V)$ an increasing function of $V$. For $V \rightarrow \infty$, Eq. (2) leads to a $\delta$-function centered at $m=m_{C}$ when $\Delta \lambda=0$. In the following, we will consider the extensive case, so that $g(V)=V$.

At the coexistence of two phases, $A\left(\lambda \leq \lambda_{0}\right)$ and $B$ $\left(\lambda \geq \lambda_{0}\right)$, we have the full probability distribution

$$
P_{V}(m)=\frac{P_{V}^{(A)}(m)+P_{V}^{(B)}(m)}{\left[F_{A}(\Delta \lambda ; V)+F_{B}(\Delta \lambda ; V)\right]},
$$

where the denominator in Eq. (3) gives the correct normalization, with

$$
F_{C}(\Delta \lambda ; V)=\sqrt{\chi_{C}} \exp \left[V\left(\Delta \lambda m_{C}+\frac{\chi_{C}}{2} \Delta \lambda^{2}\right)\right] .
$$

It is simple to analytically calculate any moment of the order parameter $m$, defined as

$$
\left\langle m^{n}\right\rangle_{V}=\int_{-\infty}^{+\infty} d m m^{n} P_{V}(m),
$$

by means of Eqs. (22) -(44) and the formula 42

$$
\begin{aligned}
\int_{-\infty}^{+\infty} d x x^{n} \exp \left[-a x^{2}+b x\right]= & \sqrt{\frac{\pi}{a}} \exp \left[\frac{b^{2}}{4 a}\right] \sum_{k=0}^{k=n-1} \\
& \times \frac{n !}{k !(n-2 k) !} \frac{(2 b)^{n-2 k}}{(4 a)^{n-k}} .
\end{aligned}
$$

In particular, we readily obtain closed analytic expressions for (our purposes very useful) second and fourth order reduced cumulants, namely, $U_{2}=\left\langle m^{2}\right\rangle_{V} /\langle m\rangle_{V}^{2}$ and $U_{4}=1-\left\langle m^{4}\right\rangle_{V} /\left(3\left\langle m^{2}\right\rangle_{V}\right)$.

The pseudo-transition point $\lambda=\lambda_{V}$ can be estimated in different ways, such as for both phases presenting equal weights (areas) or through the maximum of the global variance $V\left(\left\langle m^{2}\right\rangle_{V}-\langle m\rangle_{V}^{2}\right)$. From the present formalism, we have shown in [12] that for $V$ large and in first order in $\left(\lambda_{V}-\lambda_{0}\right)$, one gets in both cases

$$
\lambda_{V} \approx \lambda_{0}-\frac{1}{2 V} \frac{\ln \left[\chi_{B} / \chi_{A}\right]}{\left(m_{B}-m_{A}\right)} .
$$

On the other hand, seeking for a $\lambda_{V}$ given the maximum of $U_{2}$, one finds [12]

$$
\lambda_{V} \approx \lambda_{0}-\frac{1}{2 V} \frac{\ln \left[\chi_{B} / \chi_{A}\right]+2 \ln \left[m_{B} / m_{A}\right]}{\left(m_{B}-m_{A}\right)} .
$$

As we will see, Eqs. (7) and (8) give very similar results for large $V$ 's (comparing with Eqs. (7) and (8), we should mention a small misprint in the denominator of the corresponding Eqs. in [12]). Likewise DNPT to absorbing states [12], the difference between $\lambda_{V}$ and $\lambda_{0}$ consistently scales with $1 / V$ (this is also true by calculating $\lambda_{V}-\lambda_{0}$ from the minimum of $U_{4}$, however an expression not shown here). In the following we demonstrate the usefulness of our analysis by discussing distinct models.

The ZGB model with desorption of $C O$ : As a first example, we address the Ziff-Gulari-Barshard (ZGB) model 13] with CO desorption [43, 44]. The original ZGB is a longstanding model reproducing relevant features of carbon monoxide oxidation on a catalytic surface (represented by a square lattice). Each site can be empty $(*)$ or occupied by either an oxygen, $\mathrm{O}$, or a carbon monoxide, $\mathrm{CO}$. The interaction rules are:

$$
\begin{array}{r}
\mathrm{CO}_{\text {gas }}+* \rightarrow \mathrm{CO}_{\text {ads }} \\
\mathrm{O}_{2 \text { gas }}+2 * \rightarrow 2 \mathrm{O}_{\text {ads }} \\
\mathrm{CO}_{\text {ads }}+\mathrm{O}_{\text {ads }} \rightarrow \mathrm{CO}_{2}+2 * .
\end{array}
$$

Molecules of $\mathrm{CO}\left(\mathrm{O}_{2}\right)$ reach the surface with probability $Y(1-Y)$. Whenever a $\mathrm{CO}$ molecule hits a vacant site, the site becomes occupied. At the surface, if the $\mathrm{O}_{2}$ molecule finds two vacant nearest-neighbor sites, each $\mathrm{O}$ occupies one of them. If a $\mathrm{CO}_{2}$ molecule is formed (when $\mathrm{CO}$ and $\mathrm{O}$ are neighbors), it is immediately desorbed.

In the limit of large (low) $Y$ 's, the ZGB exhibits phase transitions between active steady and absorbing states, with the surface being saturated, poisoned, by molecules of $\mathrm{CO}(\mathrm{O})$. In the former (latter) situation we have strong discontinuous (continuous, in the DP universality class) transitions. This first-order absorbing phase transition has been analyzed in Ref. [12], and in fact has attracted great theoretical interest [45].

Experimentally, however, one observes a transition from low to high densities of $\mathrm{CO}, \rho_{\mathrm{CO}}$, in the lattice, but without complete poisoning [46 48]. This is a consequence of $\mathrm{CO}$ desorption, governed by the substrate temperature. Above a certain critical temperature, $\rho_{\mathrm{CO}}$ varies smooth and there is no phase transition. The inclusion of a CO desorption rate $k$ in the initial ZGB model turns out to be very effective in describing such effect. Actually, phases of high and low $\rho_{\mathrm{CO}}$ arises only for $k$ less than some critical value $k_{c} \approx 0.039$ 43, 44]. The desorption (an extra step added to the previous set of rules) is simply implemented by randomly choosing a site, and if in it there is a $\mathrm{CO}$ molecule, then with probability $k$ the $\mathrm{CO}$ is desorbed.

We run the simulations for $t=10^{7} \mathrm{MC}$ steps and appropriate averages are evaluated after discarding the initial $10^{6} \mathrm{MC}$ steps. The results for $k=0.02$ are summarized in Fig. 1 We clearly see typical trends of a discontinuous phase transition: the variation of $\rho_{\mathrm{CO}}$ with $Y$ (inset of Fig. 1 (a)) becomes steeper as the sys- 

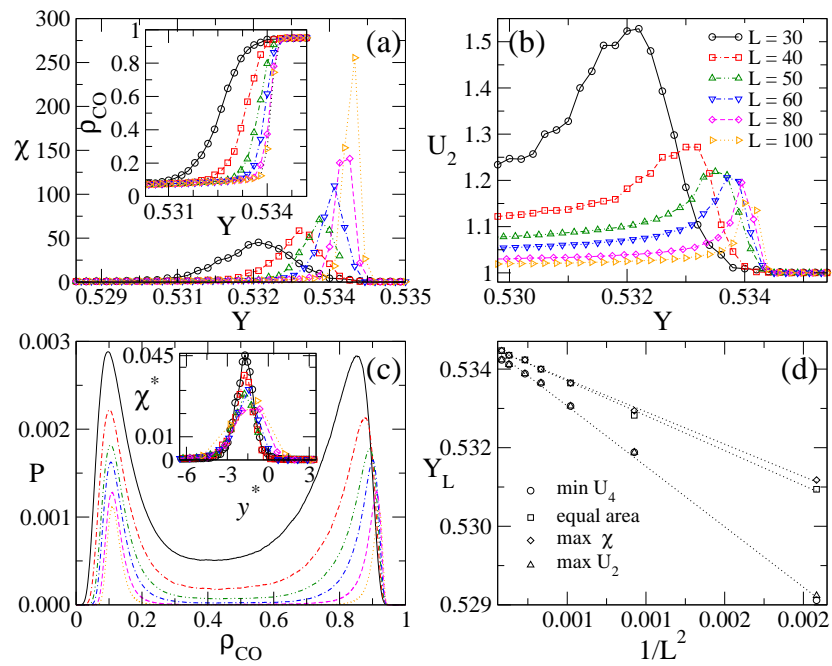

FIG. 1: (Color online) The ZGB model with desorption for distinct system sizes $L$ and $k=0.02$. (a) The order parameter variance $\chi$ (and order-parameter $\rho_{C O}$, inset) versus the creation rate $Y$. (b) The cumulant $U_{2}$ versus $Y$ and (c) the equal-area probability distribution of $\rho_{C O}$ (inset shows the data collapse by writing $\chi^{*}=$ $\chi / L^{2}$ and $\left.y^{*}=\left(Y-Y_{0}\right) L^{2}\right)$. (d) The scaling plots of $Y_{L}$ 's as function of $1 / V=L^{-2}$.

tem size $(L)$ increases, taking place in narrower intervals $\Delta Y$, which tend to agree with the maximum regions of $\chi$ (Fig. 1(a)) and $U_{2}$ (Fig. 1 (b)) for increasing $L$. Also, the overlap between the full $P_{V}\left(\rho_{\mathrm{CO}}\right)$ at the equal area condition (Fig. 1 (c)) (and as it should be expected, the minimum of $U_{4}$, but not shown here) decreases with $L$. The position of the peaks and minima are found to scale as $1 / V=L^{-2}$, from which we obtain the estimate (Fig. 1 (d)) $Y_{0}=0.5342(1)(\max . \chi), Y_{0}=0.5343(1)$ (equal area $P_{V}^{(C)}$ 's) and $Y_{0}=0.5343(1)\left(\min . U_{4}\right.$ and max. $\left.U_{2}\right)$. Note the great concordance of the different measures for $Y_{0}$. Finally, the inset in Fig. 1(c) shows a data collapse using $\chi^{*}=\chi / L^{2}$ and $y^{*}=\left(Y-Y_{0}\right) L^{2}$, confirming the correct scaling with $V=L^{2}$.

Majority vote model with inertia: The majority vote (MV) is one of the simplest nonequilibrium up-down symmetric $Z_{2}$ model exhibiting an order-disorder phase transition [49]. To each site $i$ of a network - whose vicinity is formed by its $k$ nearest neighbors - corresponds a spin variable $\sigma_{i}= \pm 1$. In the original model, with probability $1-f$ the value of $\sigma_{i}$ turns to that of the majority of the sites in the $i$ vicinity. By increasing the misalignment parameter in the interval $0 \leq f \leq 1 / 2$, the system undergoes a continuous nonequilibrium phase transition from an ordered to a disordered state 49 51].

It has been proved [50] that the continuous phase transition becomes first-order when a term depending on the local density is included in the MV dynamics, an inertial effect (for a similar mechanism in a cellular automata model, see [52]). Subsequent works have shown that even a partial inertia (i.e., for only a fraction of the sites) can
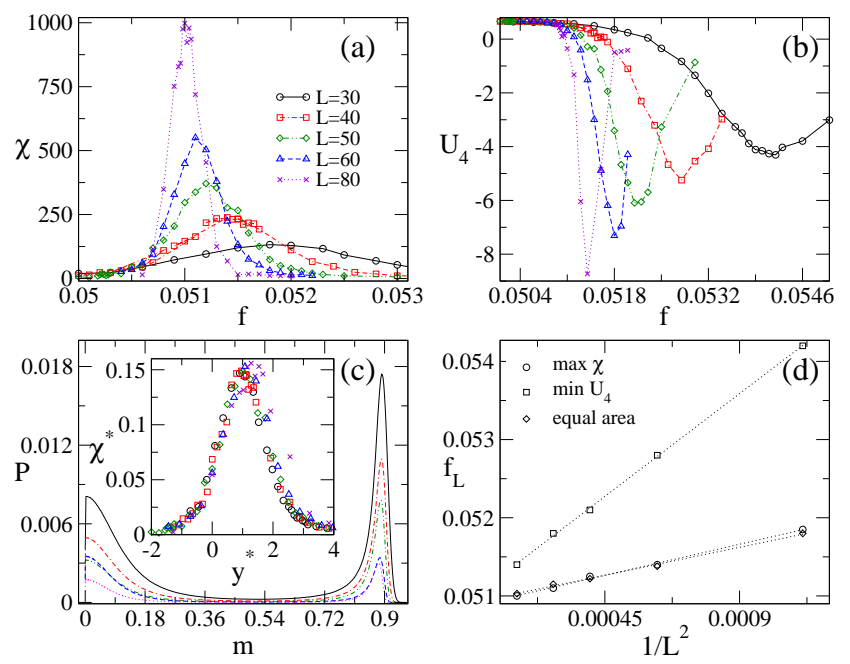

FIG. 2: (Color online) The majority vote model with inertia for distinct system sizes $L, \theta=0.375$ and $k=20$. (a) The order-parameter variance $\chi$ and (b) $U_{4}$ versus $f$. (c) The equal area probability distribution of $m$ (inset, the data collapse by writing $\chi^{*}=\chi / L^{2}$ and $\left.y=\left(f-f_{0}\right) L^{2}\right)$. (d) The scaling plots of $f_{L}$ 's versus $1 / V=L^{-2}$.

change the transition characteristic depending on the inertia strength $\theta$ [53], clarifying the necessary ingredients to originate the DNPT [54]. For low $\theta$ and $k$, the phase transition is continuous, whereas it is discontinuous for both parameters high enough.

Very recently it has been verified [54] that the same sort of continuous to discontinuous change - observed in networks - can take place in regular square lattices. At each time step, a randomly chosen $\sigma_{i}$ is flipped $\left(\sigma_{i} \rightarrow\right.$ $-\sigma_{i}$ ) according to the probability (adapted from [50] for such regular topology)

$$
w\left(\sigma_{i}\right)=\frac{1}{2}+\left(f-\frac{1}{2}\right) \sigma_{i} \operatorname{sign}\left[\frac{(1-\theta)}{k} \sum_{j=1}^{k} \sigma_{j}+\theta \sigma_{i}\right],
$$

where for a discontinuous phase transition a minimal of $k=20$ is required.

In Fig. 2 we show the simulation results for $\theta=0.375$ and $k=20$. Here also all the quantities scale with the inverse of the system volume, as observed for the maximum of $\chi$ (Fig. 2 (a)), minimum of $U_{4}$ (Fig. 2 (b)) and equal area distribution of $m$ (Fig. 2 (c)). Such quantities provide the estimates for $f_{0}$ (Fig. 2 (d)), 0.0509(1), $0.0510(1)$, and 0.0509(1), respectively. The data moreover collapse by setting $\chi^{*}=\chi / L^{2}$ and $y=\left(f-f_{0}\right) L^{2}$ (inset of Fig. 2 (c)).

Competitive contact process in bipartite sublattices: Lastly, we consider a system with competitive interactions in bipartite sublattices, introduced originally in [55]. It exhibits two types of transitions: (i) spontaneous symmetry-broken phase transitions, between two active states, and (ii) a continuous absorbing phase transition, 

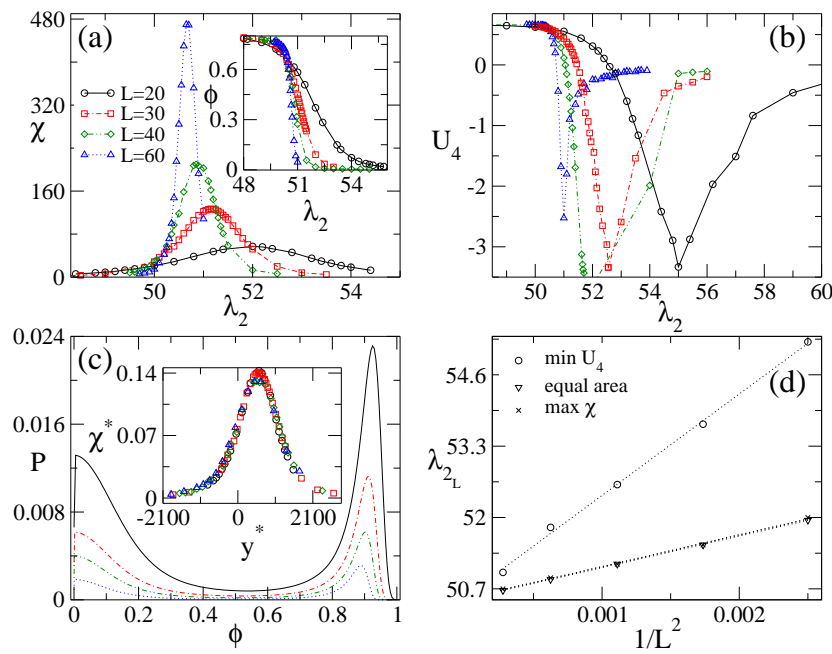

FIG. 3: (Color online) The competitive contact process in bipartite sublattices for the $a a-a s$ transition, distinct $L$ 's, $\lambda_{1}=0.5$ and $\mu=$ 1. (a) The order-parameter variance $\chi$ and the order-parameter $\phi$ (inset) versus $\lambda_{2}$. (b) The reduced cumulant $U_{4}$ versus $\lambda_{2}$. (c) The equal-area probability distribution of $\phi$ (inset, data collapse writing $\chi^{*}=\chi / L^{2}$ and $\left.y^{*}=\left(\lambda_{2}-\lambda_{20}\right) L^{2}\right)$. (d) The scaling of distinct pseudo-transition points $\left(\lambda_{2}\right)_{L}$ versus $1 / V=L^{-2}$.

whose critical behavior and universality class, DP, are not affected by the particle diffusion [56]. Nonetheless, by requiring a minimal occupied neighborhood to create a new particle (a restrictive interaction) it gives rise to distinct sorts of DNPT [57].

The model dynamics is described as the following. A particle in a given sublattice $j$ (A or B) creates autocatalitically a new particle with rate $\lambda_{1} n_{1 j} / 4\left(\lambda_{2} n_{2 j} / 4\right)$ in one of its nearest $s=1$ (next-nearest $s=2$ ) neighbor empty sites. Here, $\lambda_{s}$ is the creating strength parameter and $n_{s j}$ denotes the number of particles in the corresponding $s$ neighborhood of the considered site in sublattice $j$. A transition requires $n_{s j} \geq s$ adjacent particles (the condition $n_{1 j} \geq 1$ is similar to that of the original contact process model). This slightly modification [55] leads to the appearance of three coexistence lines, instead of critical ones. Additionally, an inhibition term depending on the local density, in the form $\mu n_{1 j}^{2}$, is included. This favors unequal sublattice populations [55]. If $\mu=0$, one recovers the traditional case in which a particle is spontaneously annihilated with rate 1 . Besides the typical absorbing (ab) and active-symmetric (as), we observe a stable active-asymmetric (aa) phase for intermediate control parameters values. The ab-as and aa-as transition lines are discontinuous (continuous) for $\lambda_{1}$ low (large). The finite size scaling for the absorbing firstorder transition has been studied in [12].

On the other hand, the aa-as line is not of absorbing type, furthermore displaying spontaneous breaking of symmetry. To locate it, we can evaluate the sublattice particle densities $\rho_{j}$. In the as phase $\rho_{A}=\rho_{B} \neq 0$, whereas in the aa phase, $\rho_{A} \neq \rho_{B}$ and $\rho=\rho_{A}+\rho_{B} \neq 0$.
Since sublattices are unequally populated in the aa phase, a natural choice for an order parameter is the difference of sublattice densities, or $\phi=\left|\rho_{A}-\rho_{B}\right|$. Unlike the as phase, in which $\phi=0$, in the aa phase $\phi \neq 0$.

In Fig. 3 (a)-(c) we show variance $\chi, U_{4}$, and equal area distribution of $\phi$, for the aa-as phase transition with $\lambda_{1}=0.5, \mu=1$ and different $L$ 's. Hence, $\lambda_{2}$ is the control parameter. Previous calculations [57] show that the symmetry-breaking transition occurs in the vicinity of $\lambda_{2}=50.96$ (evaluated for $L=80$ ). Here, the finite size analysis leads to much more precise estimations. As the previous examples, the pseudo-transition points $\left(\lambda_{2}\right)_{L}$ 's, Fig. [3(d), scale with $1 / V=L^{-2}$, from which we obtain $\left(\lambda_{2}\right)_{0}=50.55(2)$ (minimum $\left.U_{4}\right), 50.51(1)$ (equal area) and 50.53(2) (maximum $\chi$ ). Once more, the collapse (inset of Fig. 3. (c)) is very good.

Summing up, we have developed a phenomenological FSS for distinct types of DNPT, supposing only they correspond to stable steady states, for which the global balance, Eq. (1), holds true. As examples, we have considered three distinct processes: (1) transitions between low-density (high active) and high-density (low active) phases, in the context of the ZGB model with desorption; (2) order-disorder phase transitions induced by inertial effects, discussing the majority vote model with inertia; and (3) symmetry-breaking transitions between active phases, for a competitive contact process in bipartite sublattices. In all these cases, the finite-size behaviors are fully described by the present phenomenological theory.

We believe the results here derived will help to put the study and understanding of generic (nonequilibrium) discontinuous phase transition on more firm basis.

We acknowledge CNPq, CT-Infra, and Fapesp for research grants.

[1] J. Marro and R. Dickman, Nonequilibrium Phase Transitions in Lattice Models (Cambridge University Press, Cambridge, 1999).

[2] G. Ódor, Universality In Nonequilibrium Lattice Systems: Theoretical Foundations (World Scientific, Singapore, 2007)

[3] M. Henkel, H. Hinrichsen, and S. Lubeck, NonEquilibrium Phase Transitions Volume I: Absorbing Phase Transitions (Springer-Verlag, The Netherlands, 2008).

[4] H. Hinrichsen, Adv. Phys. 49, 815 (2000).

[5] G. Ódor, Rev. Mod. Phys 76, 663 (2004).

[6] R. K. Niven, Phil. Trans. R. Soc. B 365, 1323 (2010).

[7] K. A. Takeuchi, M. Kuroda, H. Chaté, and M. Sano, Phys. Rev. Lett. 99, 234503 (2007).

[8] L. Corté, P. M. Chaikin, J. P. Gollub, and D. J. Pine, Nature Physics 4, 420 (2008).

[9] S. Okuma, Y. Tsugawa, and A. Motohashi, Phys. Rev. B 83, 012503 (2011). 
[10] K. A. Takeuchi, J. Stat. Mech. 2014, P01006 (2014).

[11] C. E. Fiore, Phys. Rev. E 89, 022104 (2014).

[12] M. M. de Oliveira, M. G. E. da Luz, and C. E. Fiore, Phys. Rev. E 92, 062126 (2015).

[13] R. M. Ziff, E. Gulari, and Y. Barshad, Phys. Rev. Lett. 56, 2553 (1986).

[14] M. Ehsasi, M. Matloch, O. Frank, J. H. Block, K. Christmann, F. S. Rys, and W. Hirschwald, J. Chem. Phys. 91, 4949 (1989).

[15] M. M. de Oliveira, R. V. Santos, and R. Dickman, Phys. Rev. E 86, 011121 (2012); M. M. de Oliveira and R. Dickman, Phys. Rev. E 90, 032120 (2014).

[16] H. Weissmann and N. M. Shnerb, EPL 106, 28004 (2014).

[17] B. Néel, I. Rondini, A. Turzillo, N. Mujica, and R. Soto, Phys. Rev. E 89, 042206 (2014).

[18] G. O. Cardozo and J. F. Fontanari, Physica A 359, 478 (2006).

[19] L. Chen, F. Ghanbarnejad, W. Cai, and P. Grassberger, EPL 104, 50001 (2013).

[20] N. Crokidakis and E. Brigatti, J. Stat. Mech. P01019 (2015).

[21] C. Castellano, M. Marsili, and A. Vespignani, Phys. Rev. Lett. 85, 3536 (2000).

[22] D. Kondepudi and I. Prigogine, Modern Thermodynamics. From Heat Engines to Dissipative Structures (Wiley, Chichester, 1998).

[23] C. Jarzynski, Phys. Rev. Lett. 78, 2690 (1997).

[24] S. Lübeck, J. Stat. Phys. 123, 193 (2006).

[25] P. Grassberger, J. Stat. Mech. P01004 (2006).

[26] R. Dickman and R. Vidigal, J. Phys. A 35, 1147 (2002).

[27] M. M. de Oliveira and R. Dickman, Phys. Rev. E 71, 016129 (2005); Braz. J. Phys. 36, 685 (2006).

[28] M. E. Fisher and A. N. Berker, Phys. Rev. B 26, 2507 (1982).

[29] K. Binder, D. P. Landau, Phys. Rev. B 30, 1477 (1984).

[30] M. S. S. Challa, D. P. Landau, and K. Binder, Phys. Rev. B 34, 1841 (1986).

[31] K. Binder, Rep. Prog. Phys. 50, 783 (1987).

[32] J. Lee and J. M. Kosterlitz, Phys. Rev. B 43, 3265 (1991).

[33] C. Borgs and R. Kotecký, J. Stat. Phys. 61, 79 (1990).

[34] D. P. Landau and K. Binder, A guide to Monte Carlo Simulations in Statistical Physics, (Cambridge University Press, Cambridge, 2000).

[35] C. E. Fiore and M. G. E. da Luz, Phys. Rev. Lett. 107, 230601 (2011).

[36] D. A. Sivak and G. E. Crooks, Phys. Rev. Lett. 108, 150610 (2012).

[37] H. Hinrichsen, Physica A 369, 1 (2006); R. A. Blythe, J. Phys.: Conf. Ser. 40, 1 (2006); R. Brak, J. de Gier, and V. Rittenberg; J. Phys. A 37, 4303 (2004); R. A. Blythe and M. R. Evans, Phys. Rev. Lett. 87, 080601 (2002).

[38] P. T. Landsberg, Eur. J. Phys. 1, 31 (1980).

[39] K. Mallick, Pramana 73, 417 (2009); T. Tomé and M. J. de Oliveira Phys. Rev. Lett. 108, 020601 (2012).

[40] J. Keizer, Statistical Thermodynamics of Nonequilibrium Processes (Springer-Verlag, New York, 1987); H. Larralde and D. P. Sanders, J. Phys. A 42, 335002 (2009).

[41] Claudio Landim, Aniura Milanés, and Stefano Olla, Markov Proc. Rel. Fields 14, 165 (2008); M. Cramer, C. M. Dawson, J. Eisert, and T. J. Osborne, Phys. Rev. Lett. 100, 030602 (2008); M Cramer and J Eisert, New J. Phys. 12, 055020 (2010).

[42] E. W. Weissten, CRC Concise Encyclopedia of Mathe- matics 2nd ed. (Chapman \& Hall/CRC, Boca Raton-FL, 2003) p. 1164.

[43] B. J. Brosilow and R. M. Ziff, Phys. Rev. A 46, 4534 (1992)

[44] T. Tomé and R. Dickman, Phys. Rev. E 47, 948 (1993).

[45] M. M. de Oliveira and R. Dickman, Physica A 343, 525 (2004).

[46] P. Fisher and U. M. Titulaer, Surf. Sci. 221, 409 (1989).

[47] J. W. Evans, Langmuir 7, 2514 (1991).

[48] V. P. Zhdanov and B. Kasemo, Surf. Sci. Rep. 20, 111 (1994).

[49] M. J. de Oliveira, J. Stat. Phys. 66, 273 (1992).

[50] H. Chen, C. Shen, G. He, H. Zhang, and Z. Hou, Phys, Rev. E 91, 022816 (2015).

[51] L. F. Pereira and F. G. B. Moreira, Phys. Rev. E 71, 016123 (2005).

[52] K. Kramer, M. Koehler, C. E. Fiore, M. G. E. da Luz, Entropy 19, 102 (2017).

[53] P. E. Harunari, M. M. de Oliveira, and C. E. Fiore, Phys Rev. E 96, 042305 (2017).

[54] J. M. Encinas, P. E. Harunari, M. M. de Oliveira, and C. E. Fiore, arXiv:1802.05256

[55] M. M. de Oliveira and R. Dickman, Phys. Rev. E 84, 011125 (2011).

[56] M. M. de Oliveira and C. E. Fiore, J. Stat. Mech. 2017, 053211 (2017).

[57] S. Pianegonda and C. E. Fiore, J. Stat. Mech. 2014, P05008 (2014). 